\title{
A Wide Dynamic Range CMOS Laser Radar Receiver With a Time-Domain Walk Error Compensation Scheme
}

\author{
S. Kurtti, J. Nissinen, and J. Kostamovaara, Senior Member, IEEE
}

\begin{abstract}
This integrated receiver channel designed for a pulsed time-of-flight (TOF) laser rangefinder consists of a fully differential transimpedance amplifier channel and a timing discriminator. The amplitude-dependent timing walk error is compensated by measuring the width and rise time of the received pulse echo and using this information for calibration. The measured bandwidth, transimpedance and minimum detectable signal $(\mathrm{SNR} \sim 10)$ of the receiver channel are $230 \mathrm{MHz}, 100 \mathrm{k} \Omega$ and $\sim 1 \mu \mathrm{A}$ respectively. The single-shot precision of the receiver is $\sim 3 \mathrm{~cm}$ at an SNR of 13 and the measurement accuracy is $\pm 4 \mathrm{~mm}$ with compensation within a dynamic range of $\sim 1: 100000$. The receiver circuit was realized in a $0.35 \mu \mathrm{m}$ CMOS process and has a power consumption of $150 \mathrm{~mW}$. The functionality of the receiver channel was verified over a temperature range of $-20{ }^{\circ} \mathrm{C}$ to $+50{ }^{\circ} \mathrm{C}$.
\end{abstract}

Index Terms-Laser radar receiver, laser rangefinder, lidar, timing discrimination.

\section{INTRODUCTION}

$\mathbf{L}$ ASER ranging is widely used for various distance measurement applications, e.g., for measuring the large-scale geometry of distant natural objects, level heights in silos and containers and for various uses in geodesy and forestry [1]-[3]. Laser ranging is usually based on time-of-flight (TOF) techniques, in which the transit time $(\Delta T)$ of a laser pulse to the target and back to the receiver is measured. Since the velocity of light $(c)$ is well-known and relatively stable under varying environmental conditions, the distance to the target can be calculated from the time lapse. In applications which are not critical with regard to the permitted measurement time, continuous wave (CW) laser modulation is typically used, whereupon the transit time is measured from the phase difference between the transmitted and received laser beams. On the other hand, use of the pulsed TOF principle enables one to achieve a high measurement speed, since even a measurement based on a single transmitted pulse may give $\mathrm{cm}$-level precision [4]-[6]. The pulsed TOF principle is nevertheless based on the use of short, usually ns-scale laser pulses, and thus accurate, wide-band signal processing techniques are required.

Manuscript received June 29, 2016; revised September 22, 2016; accepted October 11, 2016. This work was supported by the Academy of Finland (Centre of Excellence in Laser Scanning Research, contract no. 272196, and contract no. 255359, 263705 and 251571). This paper was recommended by Associate Editor V. J. Koomson.

The authors are with the Circuits and Systems Research Unit, University of Oulu, 90014 Oulu, Finland (e-mail: sami.kurtti@ee.oulu.fi; jan.nissinen@ee.oulu.fi; juha.kostamovaara@ee.oulu.fi).

Color versions of one or more of the figures in this paper are available online at http://ieeexplore.iee.org.

Digital Object Identifier 10.1109/TCSI.2016.2619762

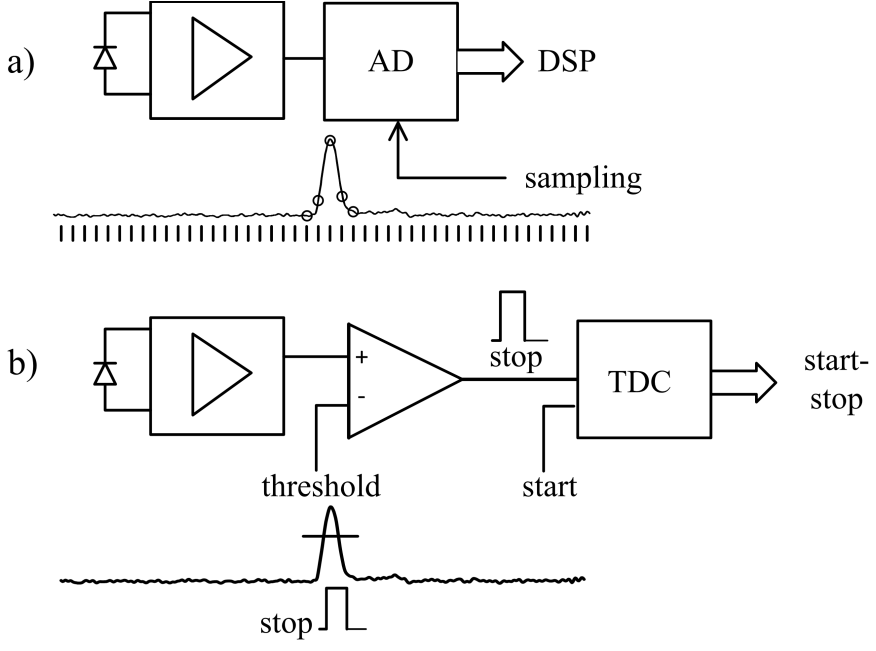

Fig. 1. (a) Sampling based approach, (b) event-based timing approach.

Pulsed time-of-flight laser radar techniques have recently shown a potential for use in 3-D imaging and environment perception systems in general, which have applications in electrically-assisted driving, in the programming of robots and drones and in gesture control, for example [7], [8]. One successful realization architecture utilizes a multi-channel spinning radar unit that can simultaneously measure distances on several, e.g., 16 or 32, vertical planes [9].

The targets in the 3-D applications are typically noncooperative and the measurement range is typically a few tens of meters, while the required single-shot precision is at the $\mathrm{cm}$ level. This performance level can be achieved with laser pulses having a peak power and pulse width of $10 \ldots 30 \mathrm{~W}$ and $3 \ldots . .5 \mathrm{~ns}$, respectively [1], [6]. Pulses of this kind can easily be produced with a semiconductor laser diode (LD)-based transmitter, while the typical of realization of the receiver part includes an avalanche photo diode (APD) optical detector, a low-noise pre-amplifier, post-amplifiers and a multi-bit ADconverter, see Fig. 1(a). Since ns-scale pulses are being used, the output of the receiver is typically sampled at a high rate of more than $200 \mathrm{Ms} / \mathrm{s}$. The high pulsing rate and the need for the multi-bit AD-converter raise the issue of high power consumption (W-range), however, which is emphasized in multi-channel realizations [3].

Another approach for the receiver is to use event-based timing, Fig. 1(b). In this architecture the AD-converter is replaced with a non-clocked timing comparator and a time-to-digital converter (TDC). The timing comparator is triggered only 
when the received pulse exceeds a predetermined threshold, which is related to the noise level of the receiver [1], [5], [6]. A TDC is then used to measure the time interval between the emitted and received optical pulses. As a result, no continuous sampling of the receiver channel is needed, and thus the power consumption of the receiver can be substantially reduced. However, since multiple accurate samples of the received echo pulse are no longer available, variation in the amplitude of the echo may pose a timing accuracy problem. The amplitude of the echo varies with the distance $\left(\sim 1 / R^{2}\right)$, orientation and reflection properties of the object. The total variation may be up to 1:10000 or more depending on the application environment. In other words, the APD may give a pulse current of $1 \mu \mathrm{A}$ for a weak echo while the peak current for a strong one may be in the region of $\sim 10 \mathrm{~mA}$. The key challenge is to be able to detect the time position of the received ns-scale echo pulse accurately, with a minimum timing walk of $1 \mathrm{~cm}(\sim 70 \mathrm{ps})$, within the required dynamic range.

An example of a receiver channel in which the eventbased timing approach has been utilized is presented in [10], although the accurate timing discrimination used in that case was based on linear signal processing, which requires that the signal should remain in the linear range of the receiver channel before timing detection and should thus be undistorted in the pre-amplifier and post-amplifier of the receiver channel. In modern IC processes where the supply voltage is low, the linear range of the receiver channel is rather narrow $(1: 10 \ldots 100)$. As a result, this detection principle requires an automatic gain control (AGC) structure to keep the signal in the linear range of the receiver channel. For example, the amplitude of the optical echo can be controlled optically with a neutral density filter, although this is often slow and bulky. Electrical gain control structures [10] have also been used but they are typically incapable of handling the full dynamic range of optical signals ( $>1$ : 10000) accurately.

We present here a CMOS laser radar receiver that utilizes the event-based timing approach, in which the timing discrimination is based on the measurement of two separate time domain parameters from the echo pulse, as described in detail in the following sections. The main advantage of this approach is that high sensitivity and accuracy ( $<50 \mathrm{ps})$ can be achieved over a wide dynamic range of $>1: 10000$ even within the amplitude range in which the receiver is saturated, so that the pulse shape becomes distorted.

The paper is organized as follows. The suggested principle is described in detail and compared with alternative approaches in Section II, while Section III describes the CMOS receiver channel realization developed here, including all the blocks of the receiver. Measurement results are given and analyzed in Section IV, and finally, Section V presents a discussion and the conclusions reached.

\section{RECEIVER ARCHITECTURE}

A block diagram of the pulsed time-of-light laser radar is presented in Fig. 2. In this kind of laser radar aiming at a $\mathrm{mm}-\mathrm{cm}$ level of accuracy within a range of several tens of meters to non-cooperative targets at high measurement rates

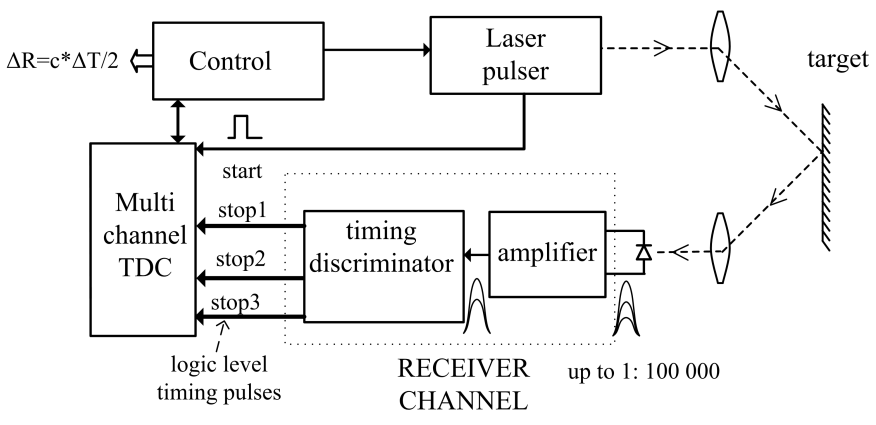

Fig. 2. Block diagram of the pulsed TOF laser rangefinder.

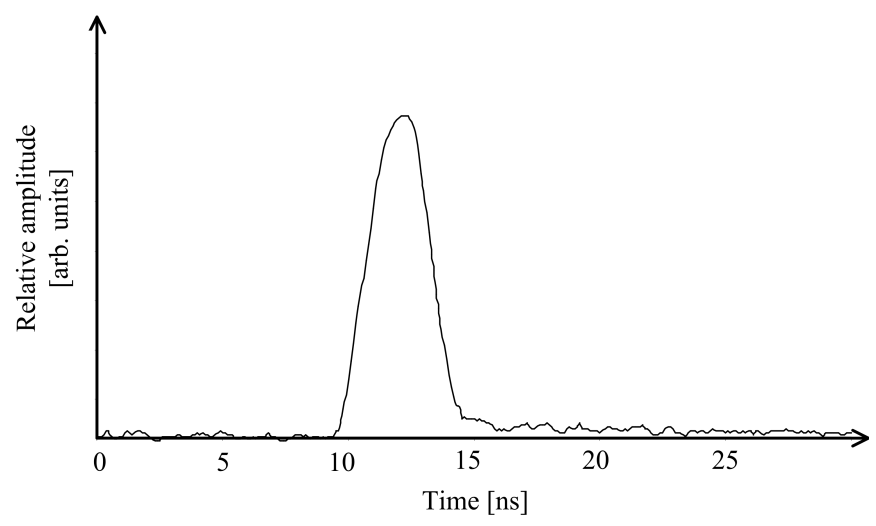

Fig. 3. Shape of the laser pulse used in rangefinding (PGAY1S06).

of $10 \ldots 100 \mathrm{kHz}$, the optical pulse is typically produced by driving a high-power laser diode with an avalanche or a MOS driver [11], [12]. Since a high peak power of $>10 \mathrm{~W}$ is typically needed, the corresponding driving current should be $\sim 10 \mathrm{~A}$ or more. This sets a practical limit on the width of the pulse, which is typically a few nanoseconds. The shape of the optical output of the laser diode (PerkinElmer PGAY1S06) used in the experiment below, when measured with a wideband $(700 \mathrm{MHz})$ photodetector, is shown in Fig. 3. The peak amplitude, wavelength and pulse width are $20 \mathrm{~W}, 905 \mathrm{~nm}$ and $3 \mathrm{~ns}$, respectively.

The timing discrimination principle used in the receiver is shown in Fig. 4. Two thresholds are used, $V_{t h_{-} L}$ and $V_{t h_{-} U}$. The lower threshold, $V_{t h_{-} L}$, is set according to the noise level of the receiver channel, the normal practice being to use a threshold that is $5 \ldots 7$ times the RMS-noise value according to the desired rate of false alarms. For low signal amplitudes that are within the linear range of the receiver the timing walk error can be compensated for using the measured width of the pulse echo at this lower threshold $V_{t h_{-} L}$. As is seen, the timing comparator Comp_width produces two stop signals, stop1 for the moment when the pulse exceeds the threshold and stop2 when it returns below the threshold. The time interval between these pulses, as measured with a multi-channel TDC, is used for compensating for the timing walk error on the basis of a separate calibration, see Fig. 5.

Unfortunately, the linear range of the receiver is typically much smaller than the amplitude range of the optical input echo, and thus of the range of pulse currents received from the 


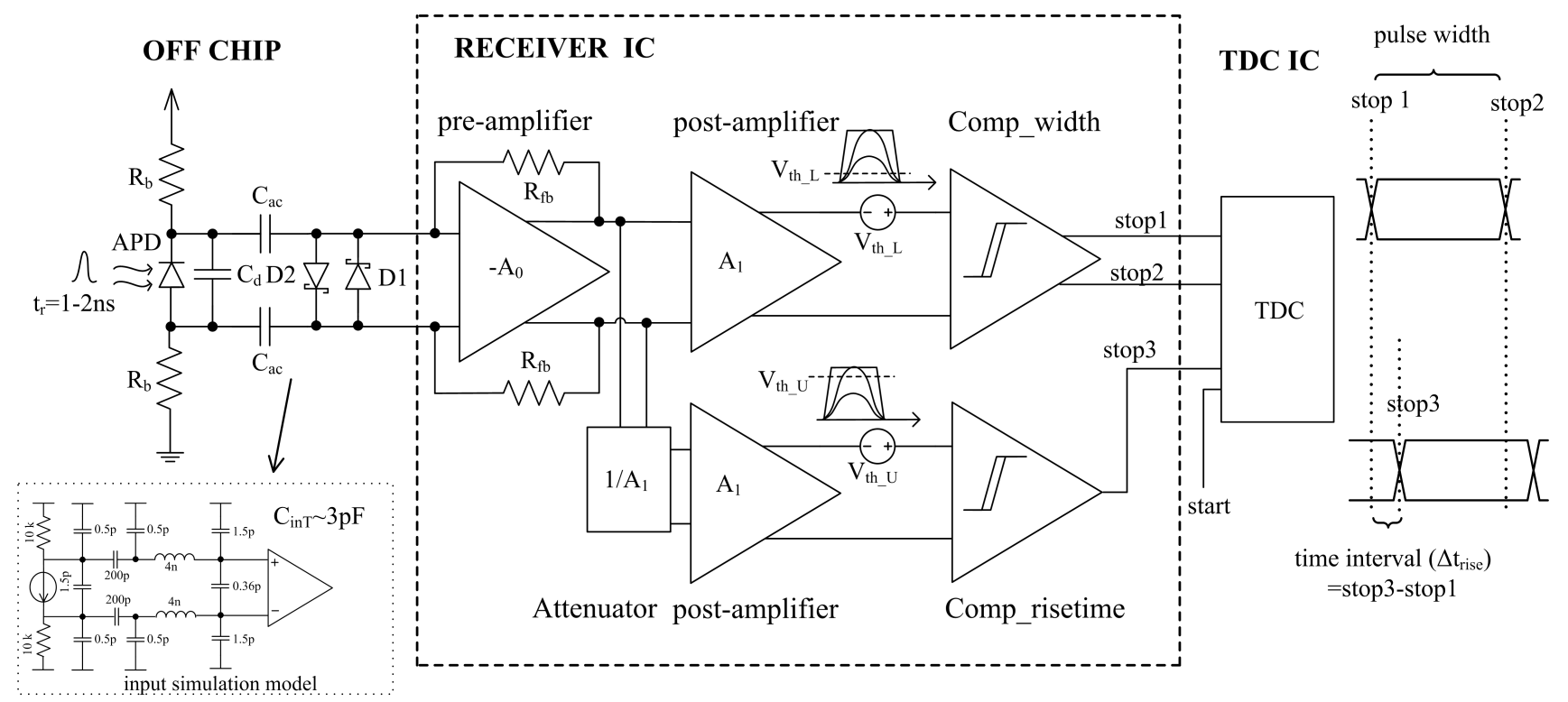

Fig. 4. Simplified block diagram of the receiver channel.
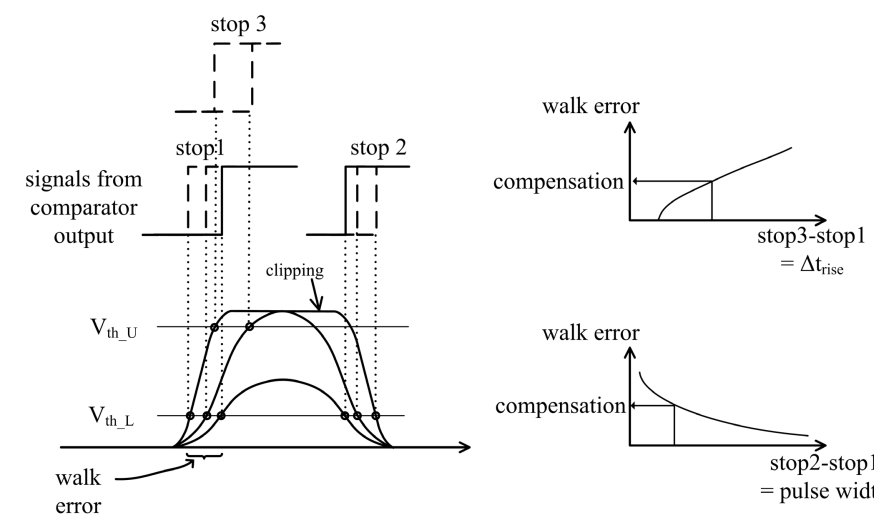

Fig. 5. Principle of timing walk compensation based on measurements of pulse width and the time interval $\Delta \mathrm{t}_{\text {rise }}$.

APD. Assume, for example, that the linear range of the transimpedance pre-amplifier is $1 \mathrm{~V}$. With a typical transimpedance of $10 \mathrm{kOhms}$, for example, the maximum signal current giving a linear response is $\sim 100 \mu \mathrm{A}$. For signal currents exceeding this limit, the pre-amplifier clips and the amplitude of the channel output is saturated. As a consequence, the measured pulse width increases well beyond the pulse width of the input, due to the integration of the extra charge into the input capacitor of the receiver. Although the measured pulse width could in principle still be used for timing walk compensation, this would be somewhat questionable, since the widening of the pulse is typically not a well-controlled phenomenon [13]. The pulse width in the saturation region depends mostly on the input circuitry of the pre-amplifier, e.g., on the operation of the protection diodes D1 and D2 and on the capacitances of the input (RC time constants), and thus the achieving of accurate compensation under varying operation conditions would be a somewhat challenging task as is shown in Section IV. So, although this principle works well within the linear range of the receiver, as has been shown in nuclear physics experiments with limited dynamic range, e.g., in [14], [15], for signals exceeding the linear range of the receiver $(\sim 100 \mu \mathrm{A} \ldots 10$ $\mathrm{mA}$ ) it may not be the optimal method.

In the receiver channel proposed here another timing comparator, Comp_risetime, with a higher threshold, $V_{t h_{-} U}$, is used to produce an additional stop signal, stop3. It is now possible to measure not only the pulse width (as in [13]) but also its rise time or, in fact, the time interval taken for the pulse to go from the lower threshold to the upper threshold $\left(\Delta t_{\text {rise }}\right)$. $\Delta t_{\text {rise }}$ can be measured even after the signal amplitude exceeds the linear range of the preamplifier. It is important to note that this time interval is measured from the linear part of the pulse (even when the pulse is eventually clipped in the receiver) and thus the somewhat unpredictable input saturation effects do not affect its value. For practical reasons, in order to have a wider variation in the measured rise time and thus to relax the accuracy required from the TDC, the output from the pre-amplifier is conducted to the timing comparator using the upper threshold. In addition, to compensate for the delay in the post-amplifier $A_{1}$, a resistive attenuator $\left(1 / A_{1}\right)$ and a similar post-amplifier are connected in between the preamplifier and the timing comparator. In conclusion, timing walk error compensation can now be accurately and repeatably achieved within the whole dynamic range of the input signal (i.e., 1:10000). It should also be noted, that while this rise time-based compensation overcomes the saturation problem, it is not in itself an optimum timing discrimination method, since it also requires that the pulse should exceed the upper threshold. Thus, from the maximum sensitivity point of view a combination of the techniques (pulse width and rise time measurement) should be used, as also shown in Fig. 5.

\section{ReCEIVER ChANNEL}

A simplified block diagram of the receiver channel is shown in Fig. 4. Fully differential structures have been used at all 
stages to reduce the effect of disturbances in the receiver channel. The optical input pulse is first converted to a current signal in an external photodetector, which is typically an avalanche photodiode (APD) in TOF applications. After that, the current signal is converted and amplified to a voltage pulse in a transimpedance-type pre-amplifier and further amplified in the post-amplifiers. Finally the timing marks (stop1, stop2 and stop3) are discriminated by means of two parallel comparators, Comp_width and Comp_risetime. The multi-channel TDC [16] has separate channels for measuring simultaneously from a single pulse the time intervals stop1-start (used to calculate the distance from the target), stop2-start (used to calculate the pulse width (stop2-stop1) for timing walk compensation) and stop3-start (used to calculate the rise time of the pulse (stop3-stop1) for timing walk compensation).

A laser pulse of the shape used in this paper, as shown in Fig. 3, has a rise time of about 1.5 ns. Preserving the pulse shape before timing discrimination in the receiver channel will minimize the timing jitter at the threshold and thus give a high timing precision, as is discussed in more detail under Measurement Results in Section IV. The required bandwidth $(B W)$ of $\sim 230 \mathrm{MHz}$ for the receiver channel to preserve its rise time can be approximated by [10], [17]

$$
B W \approx \frac{0.35}{t_{r}},
$$

where $t_{r}$ is the rise time of the pulse. On the other hand, increasing the bandwidth beyond that given in (1) would increase the noise without any significant decrease in the rise time of the timing signal, thus impairing the precision.

\section{A. Front end Design Principles}

The front end often limits the performance of the whole receiver channel, and designing the front end can also involve partly conflicting requirements, such as low noise, wide bandwidth and wide dynamic range. For measuring distances from non-cooperative targets and distances of up to several tens of meters the photodetector in pulsed TOF applications is typically an APD rather than a p-i-n photodiode (responsivity $\sim 0.5 \mathrm{~A} / \mathrm{W})$. The reason is the higher responsivity of the APD $(\sim 50 \mathrm{~A} / \mathrm{W})$ due to its high internal gain. One important parameter of the pulsed TOF laser radar receiver which affects the design of the front end is the high capacitance of the avalanche photodiode $\left(\mathrm{C}_{\mathrm{d}} \approx 1.5 \mathrm{pF}\right)$, which results from the large active area needed on account of the large dimensions of a high power laser diode (active stripe typically around $150 \mu \mathrm{m})$. In addition, the front end is greatly affected by input parasitics such as bonding wires, external packaged components, I/O protection structures, stray capacitances, the capacitances of the input transistors of the pre-amplifier etc. In the CMOS process used here, for example, the parasitic capacitance due to the analogue input PAD is $\sim 1.3 \mathrm{pF}$. As a result, the total input capacitance $\left(C_{i n T}\right)$ was approximated to be $\sim 3 \mathrm{pF}$ as shown in the input simulation model of the receiver in Fig. 4.

The transimpedance pre-amplifier shown at the front end of the receiver channel in Fig. 4 includes a core amplifier and feedback resistors. Due to the large input capacitance $\left(C_{i n T}\right)$ the dominant pole is located at the input to the receiver channel. The transfer function of the pre-amplifier, assuming a wideband core amplifier, can be approximated as [18]

$$
Z(j \omega) \approx \frac{R_{f b}}{1+\frac{R_{f b} \cdot C_{i n T}}{A_{o}} \cdot j \omega},
$$

where $R_{f b}, A_{o}$ and $C_{i n T}$ are the feedback resistance, DC gain of the core amplifier and total input capacitance, respectively. The pole frequency of this transfer function should be high enough to preserve pulse edges of 1-2 ns.

The dominant noise sources in the pre-amplifier are the feedback resistors and the equivalent voltage noise of the input transistor. The equivalent input-referred noise voltage generator $u_{n T}$ of the core amplifier is often the dominant noise source when the input capacitance at the input node is large. As a result, the equivalent spectral input current noise can be approximated as

$$
\begin{aligned}
\overline{\bar{i}_{n i n}^{2}} & \approx \frac{4 k T}{\Delta f}+u_{n T}^{2} \cdot\left|1+j \omega \cdot R_{f b} \cdot C_{i n T}\right|^{2} \\
& \approx \frac{4 k T}{R_{f b}}+\frac{8}{3} \cdot \frac{k T}{g_{m} \cdot R_{f b}^{2}} \cdot\left|1+j \omega \cdot R_{f b} \cdot C_{i n T}\right|^{2},
\end{aligned}
$$

where $g_{m}$ is the transconductance of the input transistor of the core pre-amplifier. Other noise parameters such as 1/f noise can be neglected due to the high bandwidth, while the noise arising from the resistive gate and substrate can be minimized by layout techniques.

The first term of (3) represents the thermal noise of the feedback transistor, which is white noise and can be minimized by increasing the feedback resistance. It should be noted, however, that a higher $R_{f b}$ will result in a lower bandwidth (2). In this design, $R_{f b}$ was set at $5 \mathrm{k} \Omega$, and the reduction in the receiver bandwidth was compensated for by increasing $A_{o}$ in (2). The resulting effective transimpedance of the preamplifier will be $2^{*} R_{f b}$.

The second term in (3) represents the equivalent noise voltage of the input transistor. As can be seen from (3) there is a zero in the second term of the transfer function which causes the gain to peak at high frequencies, starting from a corner frequency of $1 /\left(2 \pi R_{f b} C_{i n T}\right)$. To minimize the noise, the transconductance $\left(g_{m}\right)$ of the input transistors should also be maximized by increasing W/L and the bias current. However, increasing the W/L will also increase the input capacitance, and consequently the noise, due to noise peaking. As discussed in [19], there is in general an approximate optimum size for the input transistor.

The total RMS noise at the input to the timing comparator in a laser radar application will affect the precision and the false alarm rate [6]. For this reason it is important to limit the bandwidth of the core amplifier (within stability limits) and the post-amplifier so that the noise will be attenuated at high frequencies [18]. The core of the transimpedance pre-amplifier is composed of a transconductance amplifier followed by a transimpedance stage [20]-[22]. A schematic diagram of the core amplifier is shown in Fig. 6. 


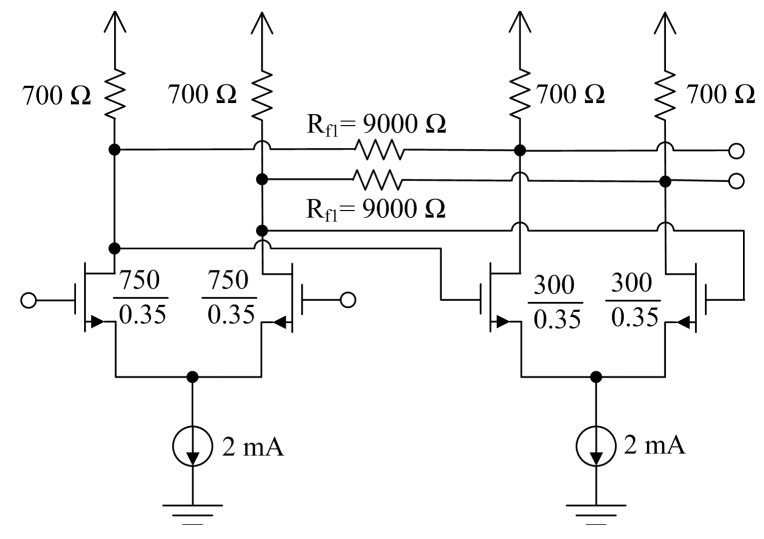

Fig. 6. Internal voltage amplifier of the transimpedance amplifier ( $A_{0}$ in Fig. 4).

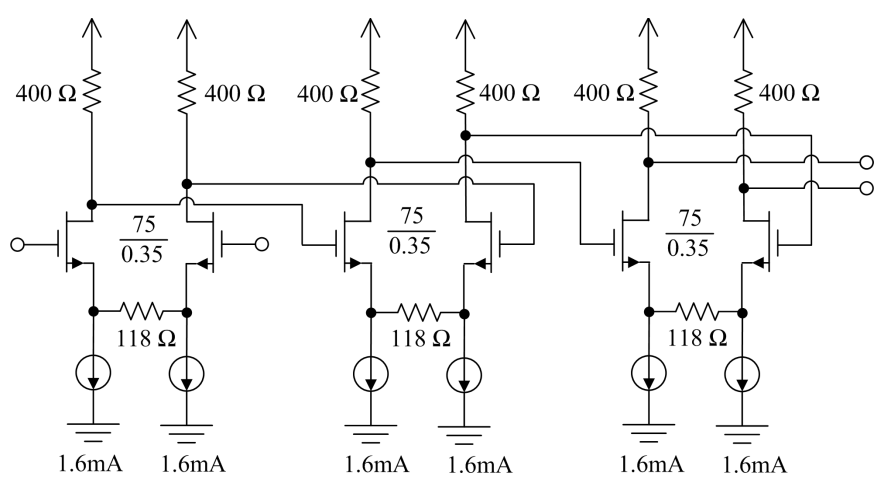

Fig. 7. Post-voltage amplifier ( $A_{1}$ in Fig. 4).

The purpose of the post-amplifier is to further amplify the output pulse of the pre-amplifier to the timing comparator. The desired minimum voltage swing $(\sim 10 \times$ RMS noise voltage $\left.\left(v_{\text {nrms }}\right)\right)$ at the input timing comparator is about $\sim 100 \mathrm{mV}$. The post-amplifier is composed of a common source (CS)coupled differential pair with source degeneration resistors. The total amplification (Atot) of the post-amplifier is $\sim 10$, and the optimum number of gain stages $(N)$ to achieve maximum GBW extension can be calculated [23] as $N=2^{*} \ln ($ Atot $)$. Three gain stages were used in the post-amplifier here, as shown in the schematic diagram in Fig. 7. A multi-stage, and thus also multi-pole, solution will also filter out high frequency noise more effectively. The resulting total transimpedance gain of the amplifier channel is $\sim 100 \mathrm{k} \Omega$.

A leading edge timing detection method is used after the post-amplifier. This will produce a systematic, amplitudedependent timing walk error [24], as shown in Fig. 5. The total timing walk error can be divided into two parts: the geometrical error $\left(t_{g}\right)$, which is generated due to the finite rise time of the laser pulse (and occurs even in an ideal receiver channel), and the walk error $\left(t_{R C}\right)$, which results from the electronic delay differences experienced by the small and large signals in the receiver channel [24], [25], the smallest signals being delayed more in proportion to the equivalent time constant $\left(R C_{e q}=1 /\left(2 \pi^{*} B W\right)\right)$. To be more specific, the total timing walk error $\left(t_{g}+t_{R C}\right)$ is approximately $\sim 2.5$ ns when the receiver channel bandwidth is $\sim 230 \mathrm{MHz}$ and the pulse rise time is $\sim 1.5 \mathrm{~ns}$, although this is compensated for by means of the proposed time domain compensation scheme.

\section{B. Timing Comparator}

The compensation principle based on pulse width and rise time measurement employed here requires two parallel timing comparators (Comp_width, Comp_risetime) each with its own threshold voltage ( $V_{t h_{-} L}$ and $\left.V_{t h_{-} U}\right)$, as shown in Fig. 4 . These timing comparators, shown in Fig. 8, consist of a resistive network for generating the thresholds, five voltage gain stages and finally a CMOS level shifter (not shown in Fig. 8) which produces timing stop signals for the TDC. The resistive networks are driven by two separate on-chip 6-bit digital-toanalogue converters (DACs) and the reference voltages are set by adjusting the currents $I_{D A C_{-} L}$ and $I_{D A C_{-} U}$. The timing comparators operate in a non-clocked configuration. Several low voltage swings, i.e., wideband gain stages, are used to maximize the gain-bandwidth product [18]. As a result, the timing walk error that is generated in the timing comparator is negligible compared with that generated at the front end.

\section{Measurement Results}

\section{A. Measurement Environment}

A block diagram of the receiver channel with $\mathrm{I} / \mathrm{O}$ pads is shown in Fig. 9. The channel is programmed by means of a SPI bus which controls the Serial-In Parallel-Out (SIPO) register. The bias circuitry in the receiver IC includes a SIPO register, biases (constant $\mathrm{gm}$ and zero temperature coefficient (TC) current generator) and control blocks. The threshold voltages of the comparators (Comp_width and Comp_risetime) can be adjusted in a range of $0-173 \mathrm{mV}\left(V_{t h_{-} L}\right)$ and 0-917 $\mathrm{mV}\left(V_{t h_{U} U}\right)$, respectively, and the receiver channel also includes threshold readout circuitry, so that $V_{t h_{-} L}$ and $V_{t h \_} U$ can also be measured separately. An analogue output buffer (OB) is used to examine the shape of the analogue timing pulse at the input to the timing comparator, and to measure the RMS noise voltage and bandwidth of the receiver channel. To increase testability, the various blocks (frontend, analogue output buffer, comparators) can be enabled or disabled separately in the receiver channel. In the default state the front end and the comparators are enabled and the analogue output buffer is disabled.

A photograph of the receiver channel, including frontend (pre-amplifier, attenuator, post-amplifiers), bias circuitry and two parallel timing comparators (Comp_width and Comp_risetime) and fabricated in a standard $0.35 \mu \mathrm{m}$ CMOS technology is shown in Fig. 10. The cells are laid out symmetrically and placed in straight rows to maximize the distance between the sensitive front-end and the noisy digital output. Shielding guard rings are used around sensitive parts such as the analogue front end and disturbing parts such as the timing comparator and digital output. In addition, one I/O pad is used as a ground ring to shield the bias, the analogue frontend and the timing comparators. The size of the layout of the receiver channel, including the pad ring, is approximately $2000 \mu \mathrm{m} \times 2000 \mu \mathrm{m}$, and the whole block was enclosed in 


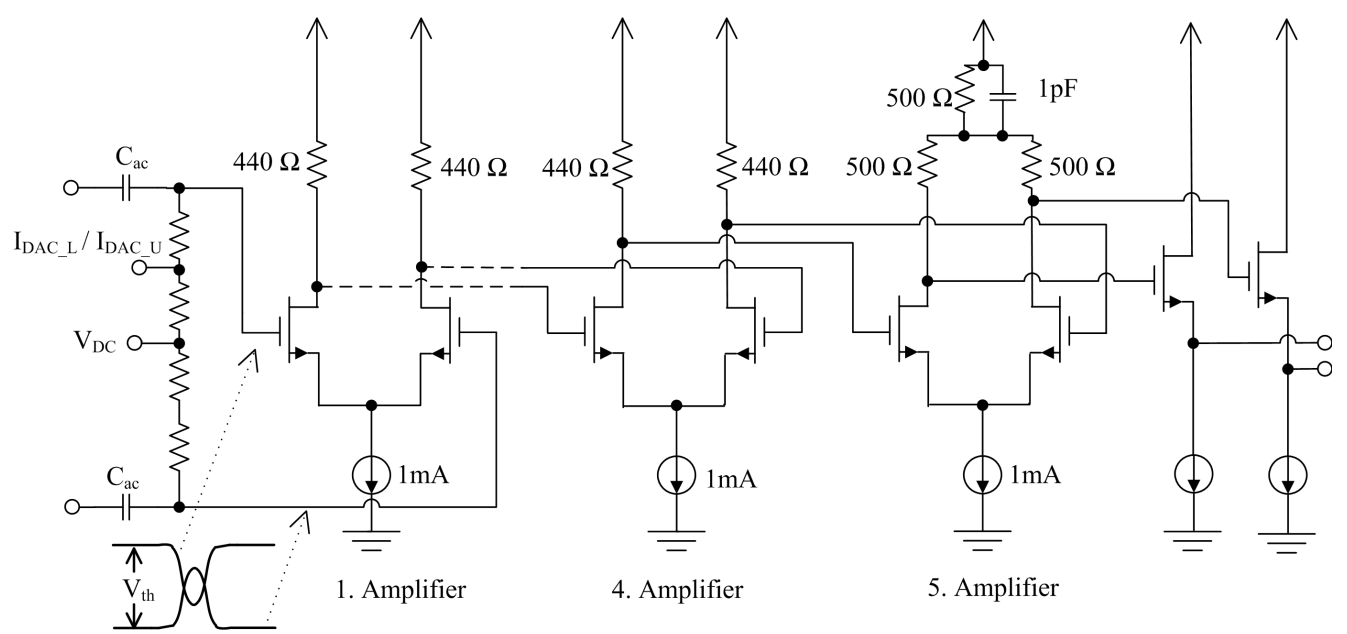

Fig. 8. Schematic diagram of the timing comparator used as Comp_width and Comp_risetime.

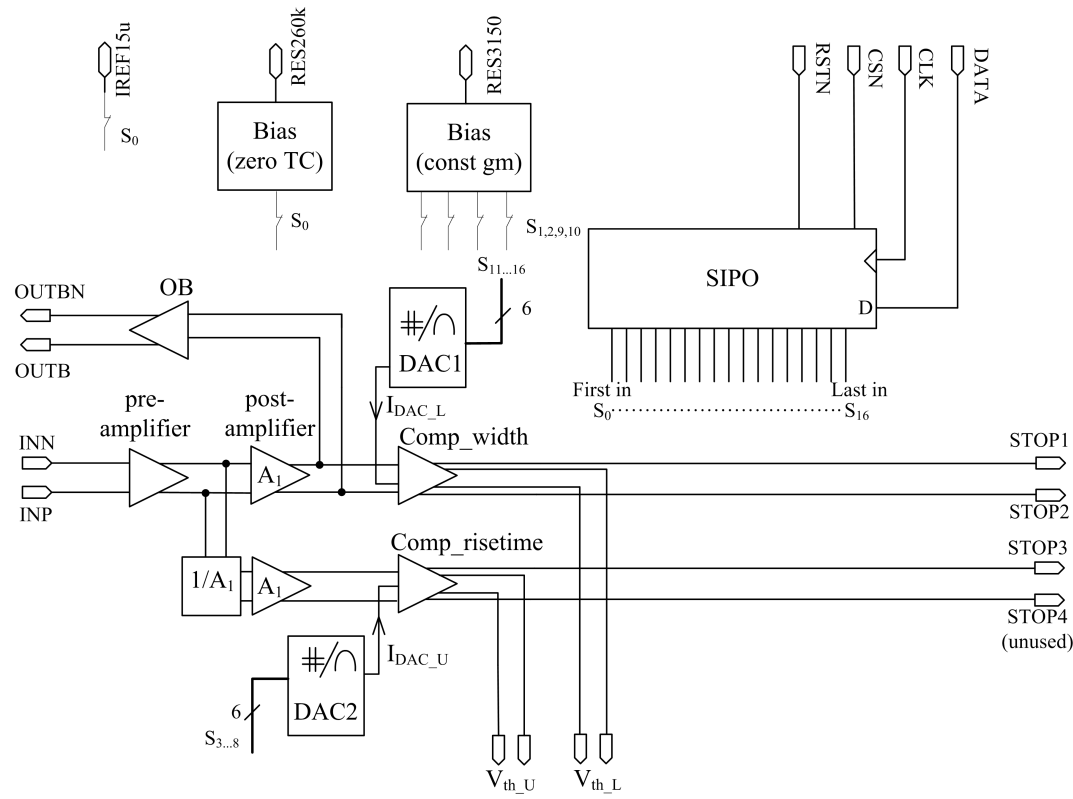

Fig. 9. Block diagram of the receiver channel with I/O pads.

a QFN36 package. Separate power supplies are used for the different IC blocks. The power consumption of the receiver chip is about $180 \mathrm{~mW}$ from a $3.3-\mathrm{V}$ supply. Measurements were made on two packaged receiver circuits.

The measurement setup is shown in Fig. 11. The receiver channel and 10-channel TDC were controlled using an Opal Kelly XEM6001 FPGA board. A multi-channel TDC [16], needed to measure the timing marks stop1, stop2 and stop3 accurately and simultaneously from the same pulse, is enclosed in a plastic QFN48 package. The LSB resolution of the TDC is about $\sim 10$ ps and its power consumption is $\sim 150 \mathrm{~mW}$ with a $3.3 \mathrm{~V}$ power supply. The TDC was fabricated in a standard $0.35 \mu \mathrm{m}$ CMOS process, so that in principle the receiver channel and time interval measurement unit could be fabricated on the same integrated chip. For the moment, however, a separate TDC chip was placed next to the receiver chip on the measurement PCB.
In the measurement setup in Fig. 11 the optical pulses were generated with a laser diode (PerkinElmer PGAY1S06) and were transmitted with a full width at half maximum (FWHM) of $\sim 3 \mathrm{~ns}$, as shown in Fig. 3. The optical pulse amplitude seen by the receiver was varied with a variable optical neutral density filter. This emulated the intensity of the received optical echo in a real application, which is due to variations in the reflectivity, orientation and distance of the target. An APD (AD230-8 TO52S1 from the First Sensor) with an active area of $230 \mu \mathrm{m}$ was biased with a voltage of $(\sim 140 \mathrm{~V})$ so that its internal gain was set at about 100 . As a result, the responsivity of the photodetector was about $35 \mathrm{~A} / \mathrm{W}$ at the used laser wavelength of $905 \mathrm{~nm}$.

An example of a measured analogue pulse is shown in Fig. 12(a)) where the pulse was measured after the postamplifier. The comparator threshold voltage $V_{t h} L$ used is also shown in Fig. 12(a)). As can be seen from the analogue buffer 


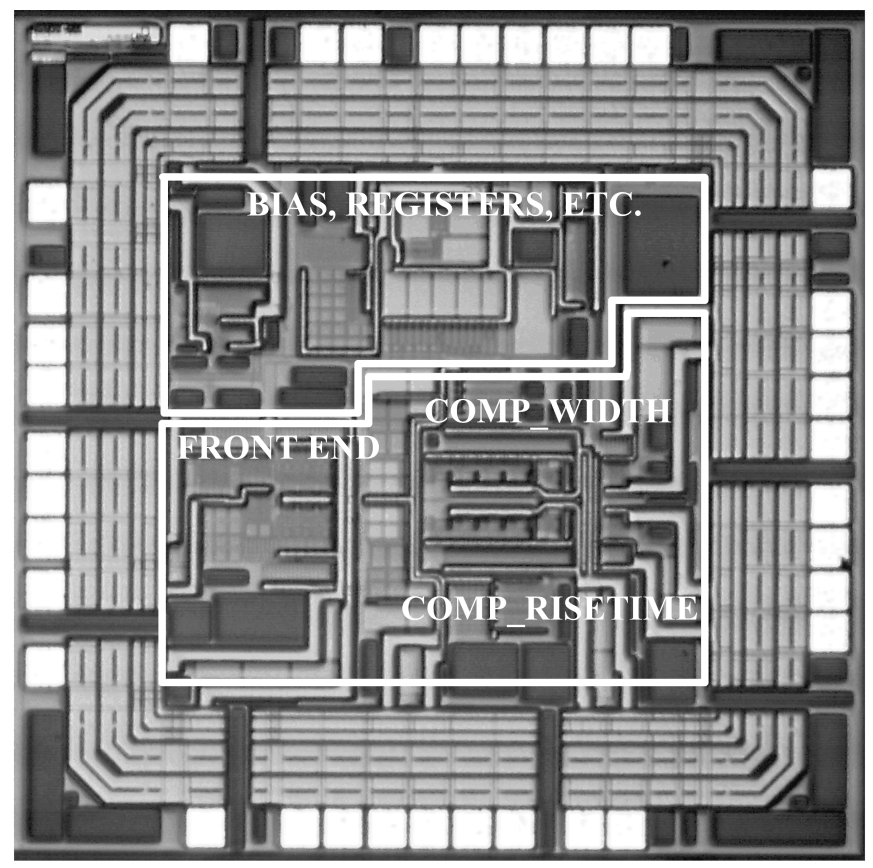

Fig. 10. Photograph of the receiver for a pulsed TOF rangefinder.

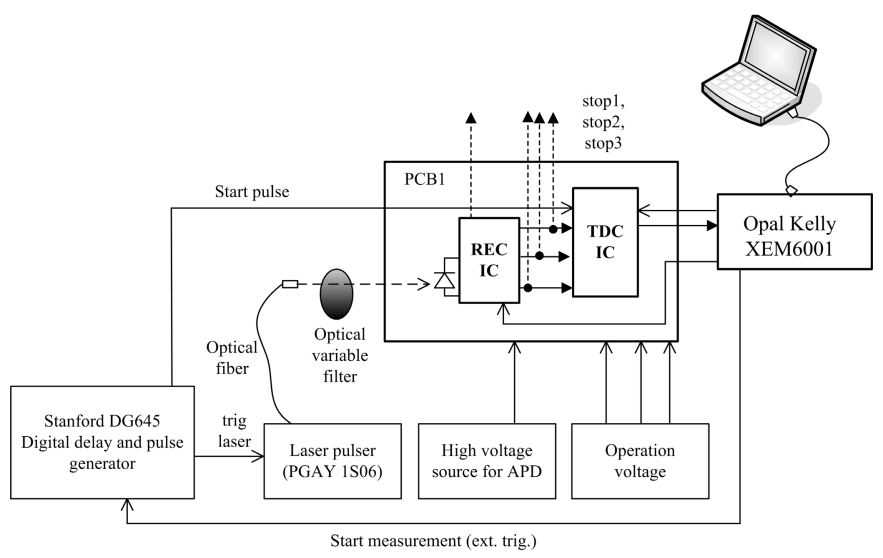

Fig. 11. Measurement setup.

pulse response, there is seen some ringing on the pulse at a frequency of $\sim 200 \mathrm{MHz}$ due to feedback from the analogue output buffer to the input of the receiver channel. The digital output of the receiver channel was examined simultaneously (stop1), as shown in Fig. 12(b)). The dashed line shows the digital response of the analogue pulse shown in Fig. 12(a)) when the analogue output buffer is enabled. In addition, the solid line in Fig. 12(b)) shows the digital output of the comparator when the analogue output buffer is disabled. It is clearly seen from the response of the comparator that disabling of analogue output buffer also interrupts the feedback between the analogue output and the input to the receiver channel. It should be noted that the analogue output buffer is always disabled in the default measurement state.
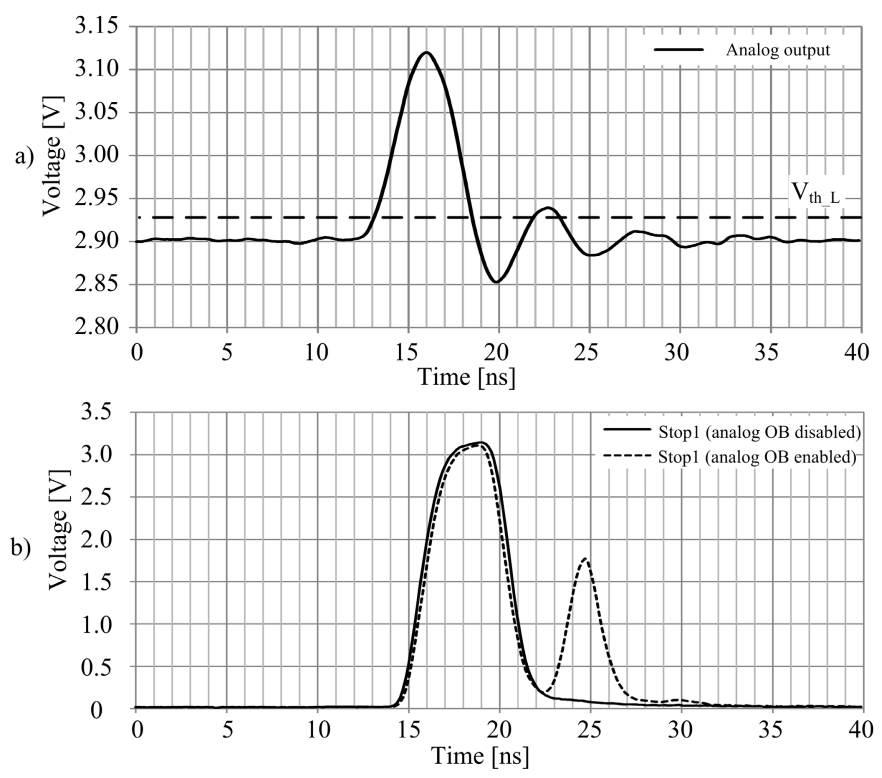

Fig. 12. (a) Analogue pulse shape, (b) Simultaneously measured output of the receiver channel (stop1).

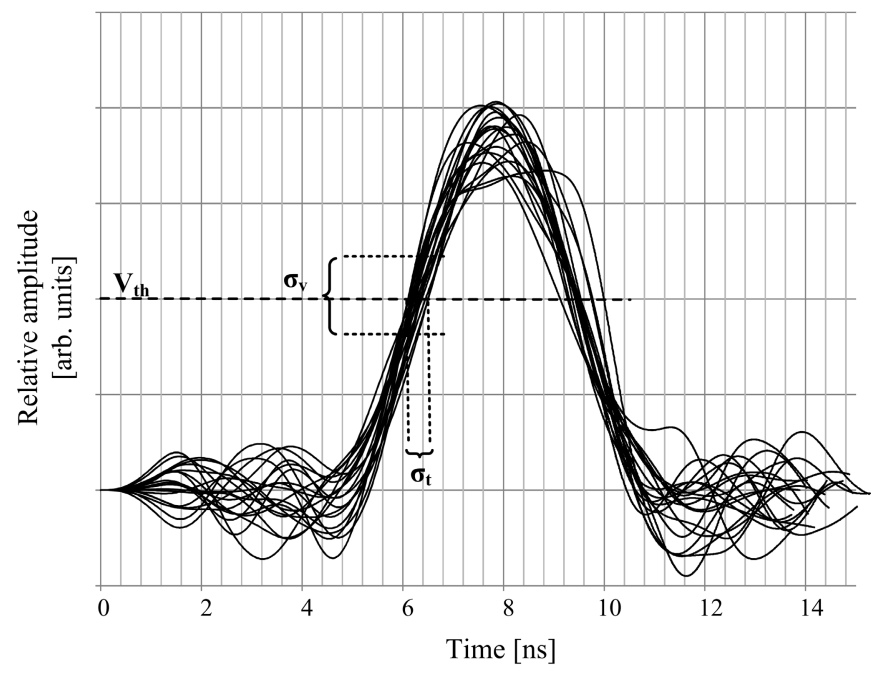

Fig. 13. Analogue timing pulses at the output of the amplifier channel, SNR $\sim 7$ (20 simulated pulses superimposed in Cadence transient noise simulations).

\section{B. Measurements of the Noise, Bandwidth and Transimpedance of the Receiver Channel}

The signal-to-noise ratio affects not only the reliability of the distance measurement (false alarm rate) but also the timing jitter. A pulsed TOF laser radar typically possesses four noise contributors: shot noise from the background illumination, noise due to the dark current in the APD, shot noise from the signal current, and finally, noise from the receiver electronics. Of these the noise due to the background illumination is usually low because of the possibility for using optical filtering, and the noise due to the dark current in the APD is negligible. Thus at a low signal level, the dominant noise is typically that due to the receiver electronics.

Random variation in the signal defines the statistical error at any given timing point due to noise, as illustrated in Fig. 13. 
The timing jitter can be approximated with the triangle rule [26]

$$
\sigma_{t}=\frac{\sigma_{v}}{\frac{d}{d t} v(t)_{t=t_{p}}},
$$

where $\sigma_{t}$ is the standard deviation of the jitter at the timing point, $\sigma_{v}$ is the standard deviation of the receiver noise and $v(t)$ is the signal at the input to the timing comparator. The noise due to the receiver electronics was measured through the jitter measurement, which is a highly important parameter in laser ranging, since it defines the precision of the system. Noise could also be measured from the analogue output, but this was now disturbed by the coupling between the analogue output and the input to the receiver. Instead, by measuring the timing jitter of single-shot measurements at a specific threshold, $V_{t h-L}(70 \mathrm{mV})$, the RMS noise voltage of the receiver electronics can be calculated based on the known slew-rate of the timing pulse. To minimize the effect on the noise measurement of the shot noise due to the signal current from the APD, the internal gain of the APD was lowered by biasing the APD to $90 \mathrm{~V}$. The slew rate was measured by setting two separate threshold voltages $V_{t h \_} L(55 \mathrm{mV}$ and $75 \mathrm{mV}$ ) for the comparator Comp_width and measuring the timing moments of the stop1 signals with respect to the thresholds. As a result a slew rate of $62 \mathrm{~V} / \mu \mathrm{s}(d / d t(v(t))$ was calculated for the timing point at a threshold voltage of $70 \mathrm{mV}$. Using equation (4), the measured timing jitter $\sigma_{t}$ of $\sim 155 \mathrm{ps}$ gives an RMS noise voltage $\sigma_{v}$ of $\sim 10 \mathrm{mV}$ for the receiver electronics.

The resulting RMS noise voltage of $10 \mathrm{mV}$ means that the threshold voltage $V_{t h_{-} L}$ of $70 \mathrm{mV}$ used here corresponds to an SNR level of $\sim 7$. The effect of the measured noise at the input to the timing comparator on the precision of the receiver is presented in more detail in section $\mathrm{E}$ below.

The 3-dB bandwidth and transimpedance of the receiver channel were measured to be $230 \mathrm{MHz}$ and $\sim 100 \mathrm{k} \Omega$, respectively, and thus the input-referred noise current of the receiver is $\sim 100 \mathrm{nA}$ when measured with the above APD detector.

\section{Timing Walk Error, Walk Compensation and Accuracy of the Receiver}

The timing walk error measurements were performed using the measurement test bench shown in Fig. 11. The lower threshold voltage $\left(V_{t h_{-} L}\right)$ for the Comp_width comparator was set to $\sim 70 \mathrm{mV}$ (SNR $\sim 7$ ) based on the noise measurements. The upper threshold voltage $\left(V_{t h_{-} U}\right)$ for the Comp_risetime comparator was set to $\sim 900 \mathrm{mV}$, which corresponds to the upper edge of the linear range of the preamplifier. The time intervals between the electrical start signal and the three timing marks stop1, stop2 and stop3 were received from the receiver channel and measured using the multi-channel TDC. The time interval start-stop1 was used to calculate an estimate for the distance from the target, and the time intervals stop3-stop1 (pulse rise time) and stop2-stop1 (pulse width) were used to compensate for the timing walk error. An electrical start was used in the timing walk error measurements and the amplitude of the input optical pulse (optical "intensity") was swept over a

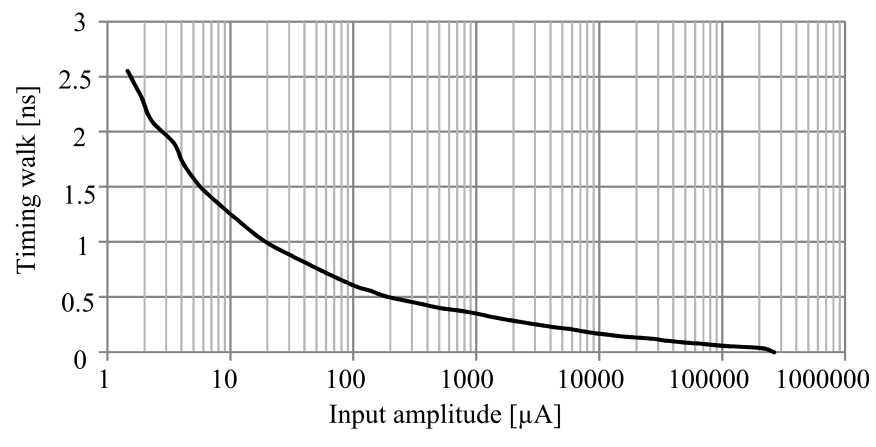

Fig. 14. Timing walk error without compensation.

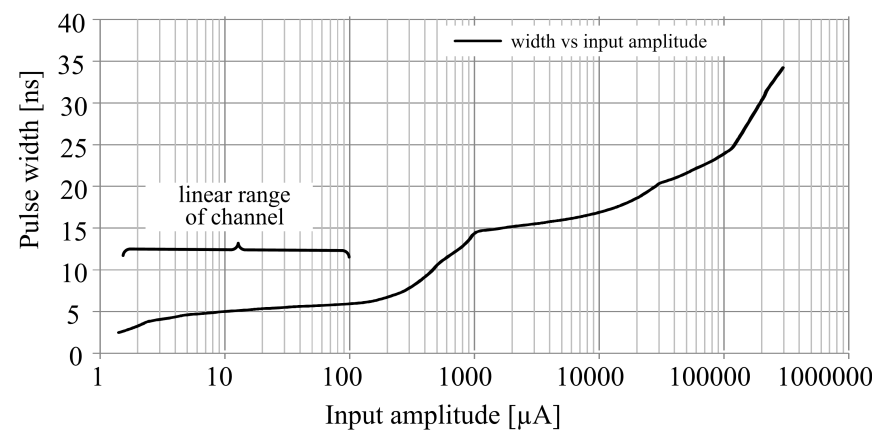

Fig. 15. Measured pulse width as a function of input amplitude.

range of $\sim 1: 100000$ with a neutral density filter. Ten thousand measurements were performed for each amplitude to achieve adequate statistical reliability.

The uncompensated timing walk error (change in the timing moment at the lower threshold value $V_{t h \_} L$ (stop1) due to the varying input amplitude) within the amplitude range $\sim 1: 100000$ is shown in Fig. 14. The timing walk error that is generated for the measured time interval (stop1-start) (distance result) is about $2.5 \mathrm{~ns}$, corresponding to a distance of $40 \mathrm{~cm}$.

The pulse width (stop2-stop1) and the time interval $\Delta t_{\text {rise }}$ (stop3-stop1) are important parameters as regards timing walk compensation. The behavior of the measured pulse width is shown in Fig. 15 as a function of the input amplitude within the dynamic region $\sim 1: 100000$. The minimum signal current of $\sim 1 \mu \mathrm{A}$ corresponds approximately to an SNR of 10. As can be seen from Fig. 15, the pulse width increases monotonously throughout the measured dynamic range, and also in the amplitude range above the linear range of the receiver channel. As a result, pulse width compensation can in principle be used for timing walk error compensation at any input amplitude. Correspondingly, the behavior of the time interval $\Delta t_{\text {rise }}$ (stop3-stop1) is shown as a function of the input amplitude in Fig. 16 within a dynamic range of $\sim 100: 100000$. Compensation based on measurement of the time interval $\Delta t_{\text {rise }}$ begins when the upper threshold voltage $V_{t h_{-} U}$ is exceeded, which corresponds to an input signal level of $\sim 100 \mu \mathrm{A}$, as can be seen in Fig. 16.

Timing walk error compensation curves can be constructed from the timing walk error measurements and the measured pulse widths (stop2-stop1) in a range of $\sim 1: 100000$, 


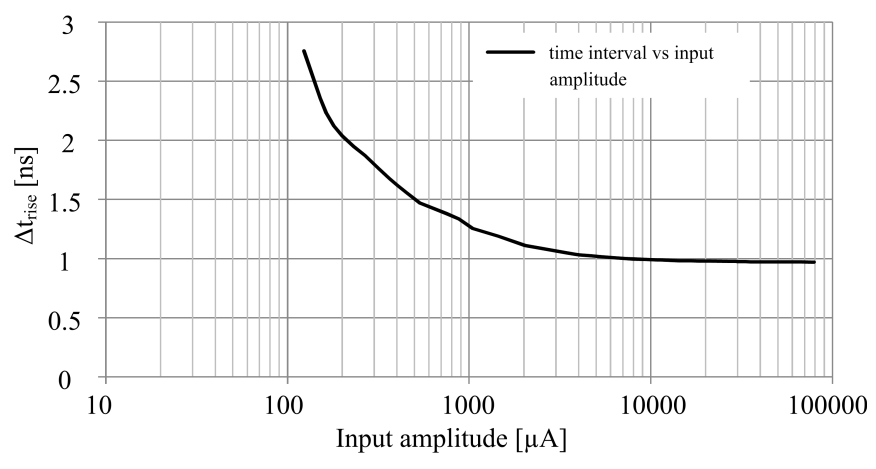

Fig. 16. Measured time interval (stop3-stop1) as a function of input amplitude.

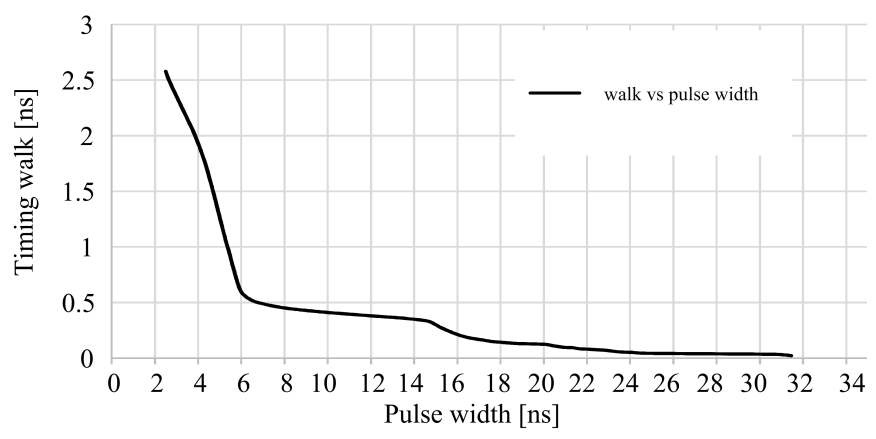

Fig. 17. Compensation curve (Walk_vs_Width).

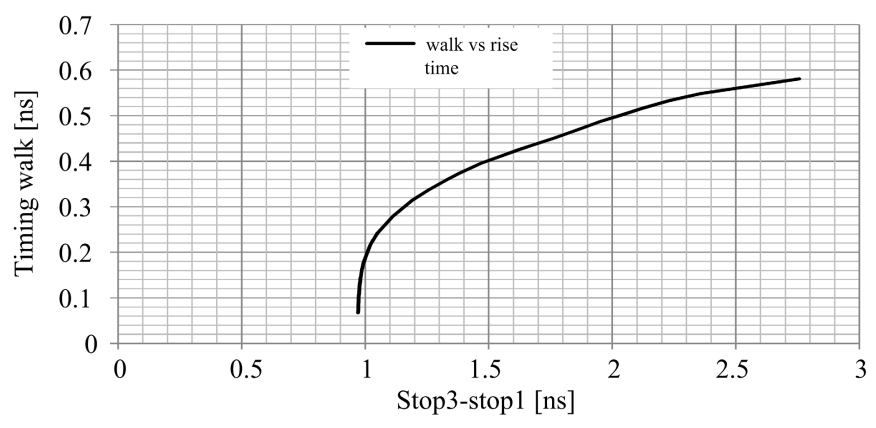

Fig. 18. Compensation curve (Walk_vs_Risetime).

and also the time interval $\Delta t_{\text {rise }}$ (stop3-stop1) in the range $\sim 100: 100000$. The measured timing walk as a function of measured pulse width (stop2-stop1) (Walk_vs_Width), over a dynamic range of $\sim 1: 100000$ containing 80 measurement points throughout the whole amplitude range is shown in Fig. 17, while the behavior of the measured timing walk error as a function of the measured time interval $\Delta t_{\text {rise }}$ (stop3-stop1) (Walk_vs_Risetime) within the dynamic range $\sim 100: 100000$ containing 46 amplitude points is shown in Fig. 18. Linear interpolations were used in both cases to achieve continuous compensation curves. The Walk_vs_Width and Walk_vs_Risetime compensation curves were stored in look up tables (LUTs) and compensation was carried out by means of these tables.

The performance of the timing walk error compensation scheme presented here was verified by measuring the residual timing walk errors of 46 amplitude points over the dynamic range of the receiver. The uncompensated walk error was first

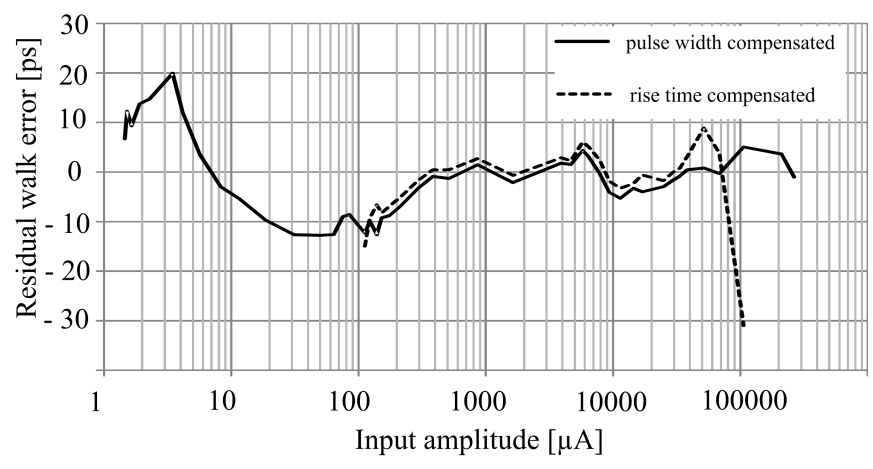

Fig. 19. Compensated timing walk errors.

measured and then compensated for by means of the curves from the look up tables. The solid line in Fig. 19 represents the residual timing walk error when compensation was based on the Walk_vs_Width compensation curve. As mentioned earlier, Walk_vs_Width compensation can be used throughout the measured dynamic range as the pulse width widens monotonously. Measured rise time information (stop3-stop1) is available above the input signal level of $\sim 100 \mu \mathrm{A}$. The dashed line in Fig. 19 represents the residual walk error when the Walk_vs_Risetime compensation curve was used. As can be seen, the residual walk error for signals exceeding the linear range of the receiver channel $(\sim 1: 100)$ is independent of the compensation principle used. The residual timing walk error shown in Fig. 19 is less than \pm 25 ps (corresponding to a distance of $\pm 4 \mathrm{~mm}$ ) over the dynamic range $\sim 1: 100000$.

The reason for a non-zero timing walk error lies partly in the quantization noise that is generated in the compensated results when compensation curves are used. The residual walk error result is also dependent on how many points have been used to extrapolate the compensation curves. At a very high input current level, saturation of the rise time, as seen in Fig. 16, causes a "tail" to appear in the compensated results in Fig. 19.

\section{Temperature Drift}

Timing walk measurements were performed over a temperature range of $-18{ }^{\circ} \mathrm{C}$ to $+52{ }^{\circ} \mathrm{C}$. In the measurement setup in Fig. 11 PCB1, which was placed in a thermal cabinet, included a receiver chip, a TDC chip and an avalanche photodiode. Uncompensated timing walk errors were measured first by sweeping the amplitude over the dynamic range of $\sim 1: 100000$ and measuring the time interval (start-stop1) at three temperatures $\left(-18{ }^{\circ} \mathrm{C}, 22{ }^{\circ} \mathrm{C}\right.$ and $\left.52{ }^{\circ} \mathrm{C}\right)$. Only Walk_vs_Width and Walk_vs_Risetime compensation curves measured at $22{ }^{\circ} \mathrm{C}$ were used for timing walk compensation at all three measurement temperatures, however.

In the temperature measurements the upper threshold $\left(V_{t h_{-} U}\right)$ was set at $\sim 0.5 \mathrm{~V}$. The reason for keeping $V_{t h \_} U$ at a lower level than the nominal linear range of the preamplifier is that the maximum swing at the output also varies (due to the constant gm biasing of pre-amplifier and post-amplifier). To be sure that rise time information was available for the timing walk compensation at every measurement temperature, 


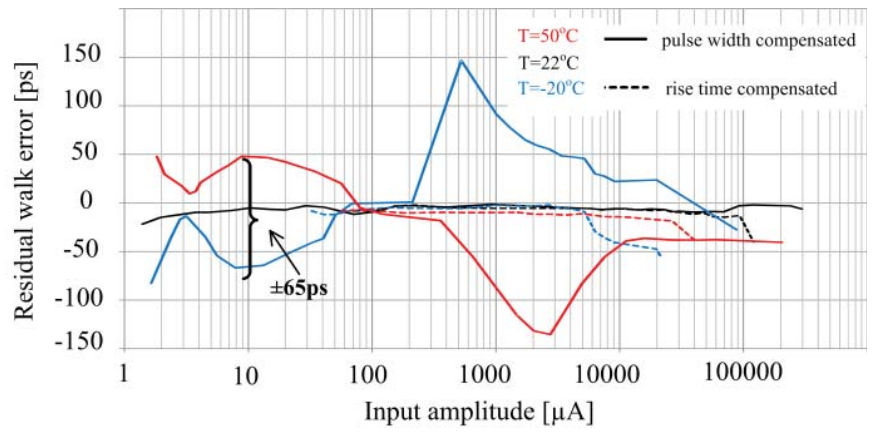

Fig. 20. Temperature dependence of the timing walk error.

the Walk_vs_Risetime compensation curve was re-measured at this $V_{t h \_} U$ value.

The residual walk errors over the dynamic range $\sim 1: 100000$ are shown in Fig. 20 at three temperatures. The solid lines show the compensated timing results when the Walk_vs_Width compensation method was used throughout the dynamic range, and correspondingly, the dashed lines denote the results when the timing walk was compensated for on the basis of rise time measurements. As can be seen from Fig. 20, when the walk compensation was based on pulse width measurement over the whole input amplitude range, the maximum residual timing error due to temperature variation was about $\pm 140 \mathrm{ps}$. It can also be seen, however, that compensation based on rise time measurements (stop3-stop1) gives significantly better results once the signal has exceeded the upper threshold voltage (input current amplitude of $\sim 100 \mu \mathrm{A}$ ) (dashed lines in Fig. 20).

The reason for this behavior is that after the linear range is exceeded and the signal is saturated in the receiver channel the measured pulse width behavior becomes more temperaturedependent (due to the receiver input parameters, for example). This is seen in Fig. 20 as an increase in the timing error of the compensated results above the input signal range of $\sim 100 \mu \mathrm{A}$ when pulse width compensation was used.

As a result the optimum method as far as walk compensation is concerned, when the measurement temperature varies is to use compensation based on the pulse width measurements over the linear range of the receiver channel and walk compensation based on rise time measurements above that range. When this is done, the maximum temperature dependence of the walk error will be about $\pm 65 \mathrm{ps}( \pm 1 \mathrm{~cm})$ over the whole dynamic region, as shown in Fig. 20.

\section{E. Single-Shot Precision}

The single-shot precision of the receiver channel is determined by the noise affecting the detection thresholds at the timing point and the precision of the TDC. In principle, the precision should improve as the input signal level is increased, but the timing jitter is also affected by the timing walk compensation used, as will be shown next.

The timing jitter of the timing mark stop1 was measured by repeating 10000 single-shot measurements at specific amplitudes and calculating the standard deviations of the time interval (start-stop1) measurements. The measured timing jitters (standard deviation, $\sigma$-value) for the rising edge (lower

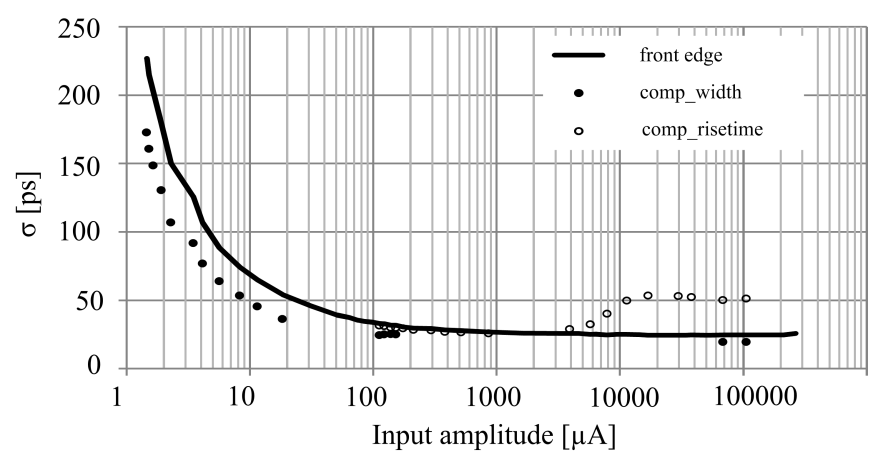

Fig. 21. Single-shot precision of the receiver.

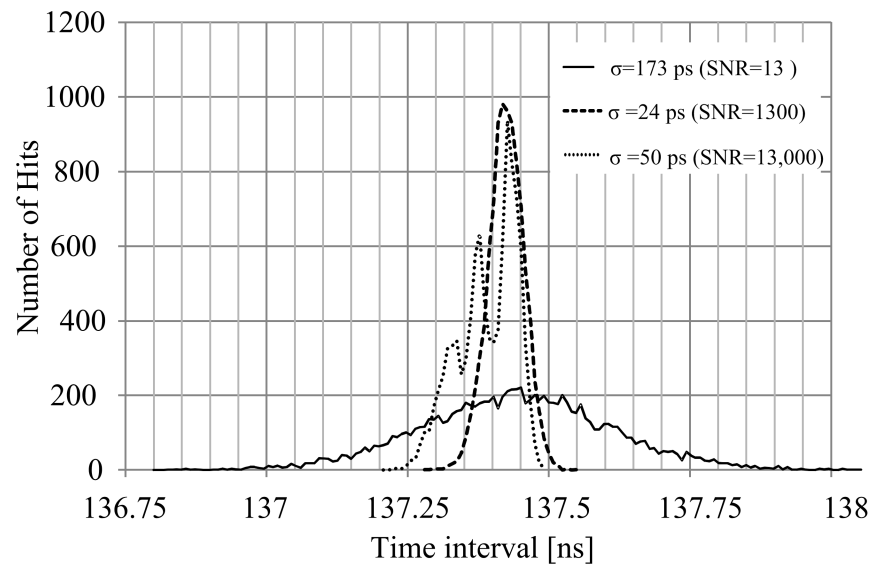

Fig. 22. Distribution of 10000 single-shot measurements of the compensated results.

threshold, $V_{t h \_L}$ ) are shown as a function of input amplitude by the solid line in Fig. 21.

After the single-shot measurement, each of the individual results was adjusted by means of the compensation curves shown in Figs. 17 and 18, which were measured during calibration. In the amplitude range below $\sim 100 \mu \mathrm{A}$, the Walk_vs_Width compensation curve (shown in Fig. 17) was used to correct the timing walk errors, while in the signal range above $\sim 100 \mu \mathrm{A}$ compensation was based on the Walk_vs_Risetime curve (shown in Fig. 18). Standard deviations for the "corrected" single-shot results were calculated from their distributions and plotted in Fig. 21.

The single-shot precision of the whole receiver, including the jitter of the TDC $(\sigma \sim 10 \mathrm{ps})$, jitter of the laser pulser, jitter induced by the compensation and the jitter of the receiver channel, was about $175 \mathrm{ps}$ (26 $\mathrm{mm}$ in distance) at an SNR level of 13. As can be seen from Fig. 21, the standard deviation of the compensated single-shot results is better than the jitter of the rising edge at low input signal levels [13], [27]. The best single shot precision achieved was approximately 20 ps at an SNR level of 1300, being limited by the measurement environment, compensation principle and the TDC. It should be noted, however, that the saturation of the rise time (stop3-stop1) shown in Fig. 18 will affect the single-shot precision of the rise time-compensated result at a very high input signal level $(>3 \mathrm{~mA})$. This is seen as an increase in the timing jitter of the compensated single- 
TABLE I

Performance Comparison of Recently Published Receivers

\begin{tabular}{|c|c|c|c|c|c|c|}
\hline & This work & [13] & [28] & [29] & [30] & [31] \\
\hline Technology & $\begin{array}{l}\text { CMOS } \\
0.35 \mu \mathrm{m}\end{array}$ & \begin{tabular}{l}
\multicolumn{1}{c}{$\mathrm{SiGe}$} \\
BiCMOS \\
$0.35 \mu \mathrm{m}$
\end{tabular} & $\begin{array}{l}\text { CMOS } \\
0.13 \mu \mathrm{m}\end{array}$ & $\begin{array}{l}\text { CMOS } \\
0.13 \mu \mathrm{m}\end{array}$ & $\begin{array}{l}\text { CMOS } \\
0.35 \mu \mathrm{m}\end{array}$ & discrete \\
\hline Bandwidth & $230 \mathrm{MHz}$ & $\sim 230 \mathrm{MHz}$ & $640 \mathrm{MHz}$ & $300 \mathrm{MHz}$ & $140 \mathrm{MHz}$ & $6 \mathrm{MHz}$ \\
\hline Type & differential & differential & single end & differential & single end & NA \\
\hline Input-referred current noise & $\sim 100 \mathrm{nA}$ & $\sim 50 \mathrm{nA}$ & $120 \mathrm{nA}$ & $100 \mathrm{nA}$ & $19 \mathrm{nA}^{*}$ & NA \\
\hline Gain control & not needed & not needed & yes & not needed & yes & not needed \\
\hline Dynamic range & $>1: 100,000$ & $1: 100,000$ & $1: 1600$ & $1: 10,000$ & $1: 12,000$ & $1: 80$ \\
\hline Accuracy@22 ${ }^{\circ} \mathrm{C}$ & $\pm 4 \mathrm{~mm}$ & $\pm 2.5 \mathrm{~mm}$ & NA & $\pm 5.5 \mathrm{~mm}$ & $\pm 210 \mathrm{~mm}$ & $\pm 3 \mathrm{~mm}$ \\
\hline Temp range & -20 to $+50^{\circ} \mathrm{C}$ & -10 to $+50{ }^{\circ} \mathrm{C}$ & -10 to $+60^{\circ} \mathrm{C}$ & -10 to $+70^{\circ} \mathrm{C}$ & -40 to $+80^{\circ} \mathrm{C}$ & NA \\
\hline Temp dependence & $\pm 10 \mathrm{~mm}$ & $\pm 18 \mathrm{~mm}$ & NA & $\pm 22 \mathrm{~mm}$ & $\begin{array}{l}\quad \pm 210 \mathrm{~mm} \text { to } \\
\pm 240 \mathrm{~mm}\end{array}$ & NA \\
\hline Precision & $\begin{array}{l}26 \mathrm{~mm} \\
@ \mathrm{SNR}=13\end{array}$ & $\begin{array}{l}18 \mathrm{~mm} \\
(a, \mathrm{SNR}=10\end{array}$ & NA & $\begin{array}{l}38 \mathrm{~mm} \\
\text { (a) } \mathrm{SNR}=20\end{array}$ & NA & $\begin{array}{cc}29 & \mathrm{~mm} \\
(a, \mathrm{SNR}=7 & \\
\end{array}$ \\
\hline
\end{tabular}

shot measurement results in Fig. 21 due to the quantization error in the rise time measurement and the steepness of the Walk_vs_Risetime compensation curve. The distributions of the timing walk-compensated 10000 single-shot measurements of three input signal levels corresponding to SNR levels of 13 (solid line), 1300 (dashed line), and 13000 (dotted line) are shown in Fig. 22. The increased timing jitter at an SNR level of $\sim 13000$ attributable to the reasons explained above is seen in Fig. 22 as a wider compensated hit distribution with quantization "spikes".

\section{Conclusions}

An integrated $0.35 \mu \mathrm{m}$ CMOS receiver channel for use in a micromodule-type pulsed time-of-flight laser rangefinder for a variety of sensor applications has been proposed, implemented and tested. A leading edge timing discriminator is used in the receiver channel, and the resulting timing walk error is compensated for by using a multi-channel time-to-digital converter to measure the rise time and the width of the received pulse and by exploiting the known relationship between these parameters and the timing walk error.

The use of time domain walk compensation enabled an accuracy of $\pm 4 \mathrm{~mm}$ (at constant temperature) to be achieved over a dynamic range of $\sim 1$ : 100000. The single-shot precision (standard deviation, $\sigma$-value) was $26 \mathrm{~mm}$ at a signal level of SNR $=13$. The measured temperature dependence of the measurement accuracy was $\sim \pm 1 \mathrm{~cm}$ over the temperature range of $-20{ }^{\circ} \mathrm{C}$ to $+50{ }^{\circ} \mathrm{C}$.

The time domain timing discrimination principle is interesting, especially since the narrow linear range of modern submicrometer IC technologies does not restrict the measurable dynamic range and automatic gain control structures are avoided. Moreover, the receiver channel and multi-channel time-to-digital converter realized here represent the same nonaggressive CMOS technology. As a result, they can in principle be integrated on the same chip.

The performance of the proposed receiver is summarized and compared with figures presented in other recently published works in this field in Table I [13], [28]-[31]. Compared to the CMOS designs, the presented receiver utilizing the pulse width/rise time timing walk error compensation method achieves the widest dynamic range with the lowest timing error. The BiCMOS receiver of [13] has comparable performance in nominal conditions but its error is almost twice larger within a narrower temperature range.

Moreover, since the timing walk error in this CMOS receiver is based on the measurement of the rise of the detected pulse at amplitudes exceeding the linear range of the receiver (and not on the measured pulse width in the saturation region), the sensitivity of the compensation is reduced to the environmental conditions (e.g., the stray capacitances of the input circuitry) of the receiver.

\section{ACKNOWLEDGEMENTS}

The authors acknowledge financial support from the Academy of Finland (Centre of Excellence in Laser Scanning Research, contract no. 272196, and contract nos. 255359, 263705, and 251571).

\section{REFERENCES}

[1] J. Kostamovaara, K. Maatta, and R. Myllyla, "Pulsed time-of-flight laser rangefinding techniques for industrial applications," Proc. SPIE, vol. 1614, pp. 283-295, Nov. 1991.

[2] A. Ullrich, N. Studnicka, J. Riegl, and S. Orlandini, "Long-range highperformance time-of-flight-based 3D imaging sensors," in Proc. Int. Symp. 3D Data Process. Visualizat. Transmiss., Padova, Italy, 2002, pp. 852-855.

[3] C. Mallet and F. Bretar, "Full-waveform topographic lidar: State-of-art," ISPRS J. Photogram. Remote Sens., vol. 64, no. 1, pp. 1-16, Jan. 2009.

[4] M.-C. Amann, T. Bosch, M. Lescure, R. Myllyla, and M. Rioux, "Laser ranging: A critical review of usual techniques for distance measurement," Opt. Eng., vol. 40, no. 1, pp. 10-19, 2001.

[5] K.-H. Thiel and A. Wehr, "Performance capabilities of laser scannersAn overview and measurement principle analysis," in Proc. ISPRS Workshop, Freiburg, Germany, Oct. 2004, pp. 14-18.

[6] H. N. Burns, C. G. Christodoulou, and G. D. Boreman, "System design of a pulsed laser rangefinder," Opt. Eng., vol. 30, no. 3, pp. 323-329, Mar. 1991.

[7] V. C. Coffey, "Imaging in 3-D: Killer apps coming soon to a device near you!" Opt. Photon. News, vol. 25, no. 6, pp. 36-43, 2014.

[8] B. Schwarz, "LIDAR: Mapping the world in 3D," Nat. Photon., vol. 4, no. 7, pp. 429-430, Jul. 2010.

[9] S. Velupillai and L. Guvench, "Laser scanner for driver-assistance systems in intelligent vehicles," IEEE Control Syst., vol. 26, no. 2, pp. 17-19, Apr. 2009. 
[10] T. Ruotsalainen, P. Palojarvi, and J. Kostamovaara, "A wide dynamic range receiver channel for a pulsed time-of-flight laser radar," IEEE J. Solid-State Circuits, vol. 36, no. 8, pp. 1228-1238, Aug. 2001.

[11] L. Hallman, J. Huikari, and J. Kostamovaara, "A high-speed/power laser transmitter for single photon imaging applications," in Proc. IEEE Sensors Conf., Valencia, Spain, Nov. 2014, pp. 1157-1160.

[12] S. N. Vainshtein, V. S. Yuferev, and J. T. Kostamovaara, "Avalanche transistor operation at extreme currents: Physical reasons for low residual voltages," Solid-State Electron., vol. 47, no. 8, pp. 1255-1263, Aug. 2003.

[13] S. Kurtti and J. Kostamovaara, "An integrated laser radar receiver channel utilizing a time-domain walk error compensation scheme," IEEE Trans. Instrum. Meas., vol. 60, no. 1, pp. 146-157, Jan. 2011.

[14] F. Anghinolfi, P. Jarron, F. Krummenacher, E. Usenko, and M. C. S. Williams, "NINO, an ultra-fast, low-power, front-end amplifier discriminator for the time-of-flight detector in ALICE experiment," in Proc. IEEE Trans. Nucl. Sci. Symp. Conf., vol. 51. Oct. 2004, pp. 1974-1978.

[15] M. Despeisse, F. Powolny, P. Jarron, and J. Lapington, "Multi-channel amplifier-discriminator for highly time-resolved detection," IEEE Trans. Nucl. Sci., vol. 58, no. 1, pp. 202-208, Feb. 2011.

[16] S. Jahromi, J. Jansson, I. Nissinen, J. Nissinen, and J. Kostamovaara, "A single chip laser radar receiver with a $9 \times 9$ SPAD detector array and a 10-channel TDC," in Proc. 41st Eur. Solid-State Circuits Conf., Graz, Austria, Sep. 2015, pp. 364-367.

[17] R. Ziemer and W. Tranter, Principles of Communications, 2nd ed. Boston, MA, USA: Houghton Mifflin, 1985.

[18] E. Sackinger, Broadband Circuits for Optical Fiber Communication. Hoboken, NJ, USA: Wiley, 2005

[19] A. A. Abidi, "On the noise optimum of gigahertz FET transimpedance amplifiers," IEEE J. Solid-State Circuits, vol. 22, no. 6, pp. 1207-1209, Dec. 1987.

[20] R. Coppoolse, J. Verbeke, P. Lambrecht, J. Codenie, and J. Vandewege, "Comparison of a bipolar and a CMOS front end in broadband optical transimpedance amplifiers," in Proc. 38th Midwest Symp. Ciruits Syst., Rio de Janeiro, Brazil, Aug. 1996, pp. 1026-1029.

[21] E. M. Cherry and D. E. Hooper, "The design of wideband transistor feedback amplifiers," Proc. IEE, vol. 110, no. 2, pp. 375-389, Feb. 1963.

[22] B. Zand, K. Phang, and D. Johns, "A transimpedance amplifier with DC-coupled differential photodiode current sensing for wireless optical communications," in Proc. Custom Integr. Circuits Conf., May 2001, pp. $455-458$.

[23] R. P. Jindal, "Gigahertz-band high-gain low-noise AGC amplifiers in fine-line NMOS," IEEE J. Solid-State Circuits, vol. 22, no. 4, pp. 512-521, Aug. 1987.

[24] P. Palojarvi, T. Ruotsalainen, and J. Kostamovaara, "A 250-MHz BiCMOS receiver channel with leading edge timing discrimination for a pulse time-of-flight laser rangefinder," IEEE J. Solid-State Circuits, vol. 40, no. 6, pp. 1341-1349, Jun. 2005.

[25] R. J. van Plassche and P. Baltus, "An 8-bit 100-MHz full-Nyquist analog-to-digital converter," IEEE J. Solid-State Circuits, vol. 23, no. 6, pp. 1334-1344, Dec. 1988.

[26] G. Bertoline and A. Coche, Semiconductor Detectors. Amsterdam, The Netherlands: North Holland, 1968

[27] L. W. Hallman and J. Kostamovaara, "Note: Detection jitter of pulsed time-of-flight lidar with dual pulse triggering," Rev. Sci. Intrum., vol. 85, no. 3, p. 036105, Apr. 2014.

[28] T.-H. Ngo, C.-H. Kim, Y. J. Kwon, J. S. Ko, D.-B. Kim, and H.-H. Park, "Wideband receiver for a three-dimensional ranging LADAR system," IEEE Trans. Circuits Syst. I, Reg. Papers, vol. 60, no. 2, pp. 448-456, Feb. 2013.

[29] J. Nissinen, I. Nissinen, and J. Kostamovaara, "Integrated receiver including both receiver channel and TDC for a pulsed time-of-flight laser rangefinder with cm-level accuracy," IEEE J. Solid-State Circuits, vol. 44, no. 5, pp. 1486-1497, May 2009.
[30] H.-S. Cho, C.-H. Kim, and S.-G. Lee, "A high-sensitivity and low-walk error LADAR receiver for military application," IEEE Trans. Circuits Syst. I, Reg. Papers, vol. 61, no. 10, pp. 3007-3015, Oct. 2014.

[31] J. Xiao, M. Lopez, X. Hu, J. Xiao, and F. Yan, "A continuous wavelet transform-based modulus maxima approach for the walk error compensation of pulsed time-of-flight laser rangefinders," Int. J. Light Electron Opt., vol. 127, no. 4, pp. 1980-1987, Feb. 2016.

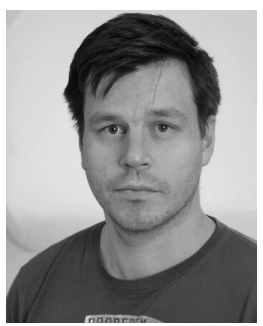

S. Kurtti was born in Kuusamo, Finland, in 1979. He received the M.Sc.Eng. and Dr.Tech. degrees in electrical engineering from the University of Oulu, Finland, in 2004 and 2013. He was a Research Scientist from 2004 to 2013 and has been a Postdoctoral Researcher with the Electronics Laboratory, University of Oulu, since 2013. His interests include the development of analogue and mixed-signal integrated circuits and structures for pulsed time-offlight laser rangefinders.

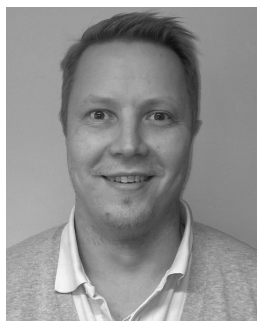

J. Nissinen was born in Oulu, Finland, in 1976. $\mathrm{He}$ received the M.Sc.Eng., Lic.Tech., and Dr.Tech. degrees in electrical engineering from the University of Oulu, Finland, in 2002, 2007, and 2011, respectively. He was a Research Scientist from 2002 to 2011, and has been a Postdoctoral Researcher with the Electronics Laboratory, University of Oulu, since 2011. His research interests include the design of analogue and mixed-signal integrated circuits for pulsed time-of-flight laser rangefinders and for pulsed

Raman spectroscopy.

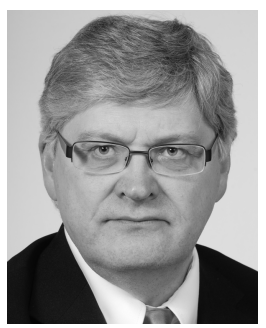

J. Kostamovaara (M'85-SM'13) was born in Tervola, Finland, in 1955. He received the Dipl.Eng., Lic.Tech, and Dr.Tech. degrees in electrical engineering from the University of Oulu, Finland, in 1980, 1982, and 1987, respectively. From 1985 to 1994 he was an Associate Professor of Electronics with the Department of Electrical Engineering, University of Oulu. In 1994, he was an Alexander von Humboldt Scholar with the Technical University of Darmstadt, Germany, after which he was invited in 1995 to become Professor of Electronics at the University of Oulu, the position which he holds now. In 2007 he was appointed to an Academy Professorship with the Academy of Finland for a term of office extending from 2006 to 2011, and later for a second term from 2012 to 2017 His current main interests are in the development of pulsed time-of-flight techniques and circuits for laser radars and optical spectroscopy. 